\title{
P-0360 - TOTUM-63 improves glucose homeostasis and reduces hepatic steatosis in mice, with or withoult metformin treatment
}

P. Sirvent, ${ }^{1,2}$, V. Chavanelle,, 2 , Y.F. Otero, ${ }^{1,2}$, S.L. Peltier ${ }^{2}$

'Clermont Auvergne University, Clermont-Ferrand, France, ${ }^{2}$ Valbiotis, La Rochelle, France

- Background: Lifestyle changes have been shown to decrease the risk of developing diabetes in prediabetic patients. However, although lifestyle changes are effective, patients rarely adhere to them on the long term. We have previously shown that the complex TOTUM-63 (T63) improves fasting glycaemia and insulin sensitivity in high fat-fed obese mice and diabetic db/db mice. Recently, a phase I clinical study showed the good tolerance and safety of T63 in human subjects. While metformin is the first line oral therapy for type 2 diabetes, the combined effects of T63 with metformin are unknown.

Aims: The aim of this study is to assess the effects of a 16-week supplementation with T63 on glucose homeostasis in high fat-fed mice, with or without concomitant metformin treatment.

- Methods: 6-weeks-old C57BL6/J male mice were fed for 16 weeks with standard diet (Control), high-fat diet (HFD), HFD + T63, HFD + metformin (MET) or HFD + T63 + MET. Food intake and weight gain were recorded weekly. Body composition, fasting blood glucose and plasma insulin levels were assessed every 4 weeks. At the end of the treatment, glucose homeostasis was evaluated with an oral glucose tolerance test (OGTT) and an insulin tolerance test (ITT). Hepatic triglyceride content was also measured at the end of the study.

- Results: T63 and Metformin did not affect food intake. Metformin treatment did not modify body weight gain in HFDfed mice. On the other hand, T63 supplementation induced a strong reduction in body weight gain $(33.6 \pm 5.9$ vs $44.7 \pm 4.6$; $\mathrm{p}<0.01$ ), which was not impacted by further addition of metformin. Similarly, body fat mass was reduced by almost 50\% following T63 supplementation $(p<0.01)$, without any significant effect with the addition of metformin. T63 slightly reduced glycaemia over the 16 weeks of supplementation, without any cumulative effect of metformin. HFD induced a marked rise in plasma insulin levels that was not altered by metformin treatment. T63 supplementation prevented the rise in plasma insulin level $(1.6+1.6$ vs $5.5 \pm 3.4 ; p<0.01)$, with or without concomitant metformin treatment. Neither 63 nor metformin improved glucose response to OGTT. However, both T63 and metformin reduced plasma insulin levels during OGTT, without any additive effect of combined treatment (Figures 1 and 2). T63 supplementation also enhanced the fall of glycaemia induced by insulin during ITT, without any additive effect of metformin treatment. Hepatic triglycerides were strongly reduced by $40 \%$ following $T 63$ supplementation in both metformin-treated and untreated mice, while metformin treatment alone did not show any effect.

Figure 1: Insulin measurements during oral glucose tolerance test

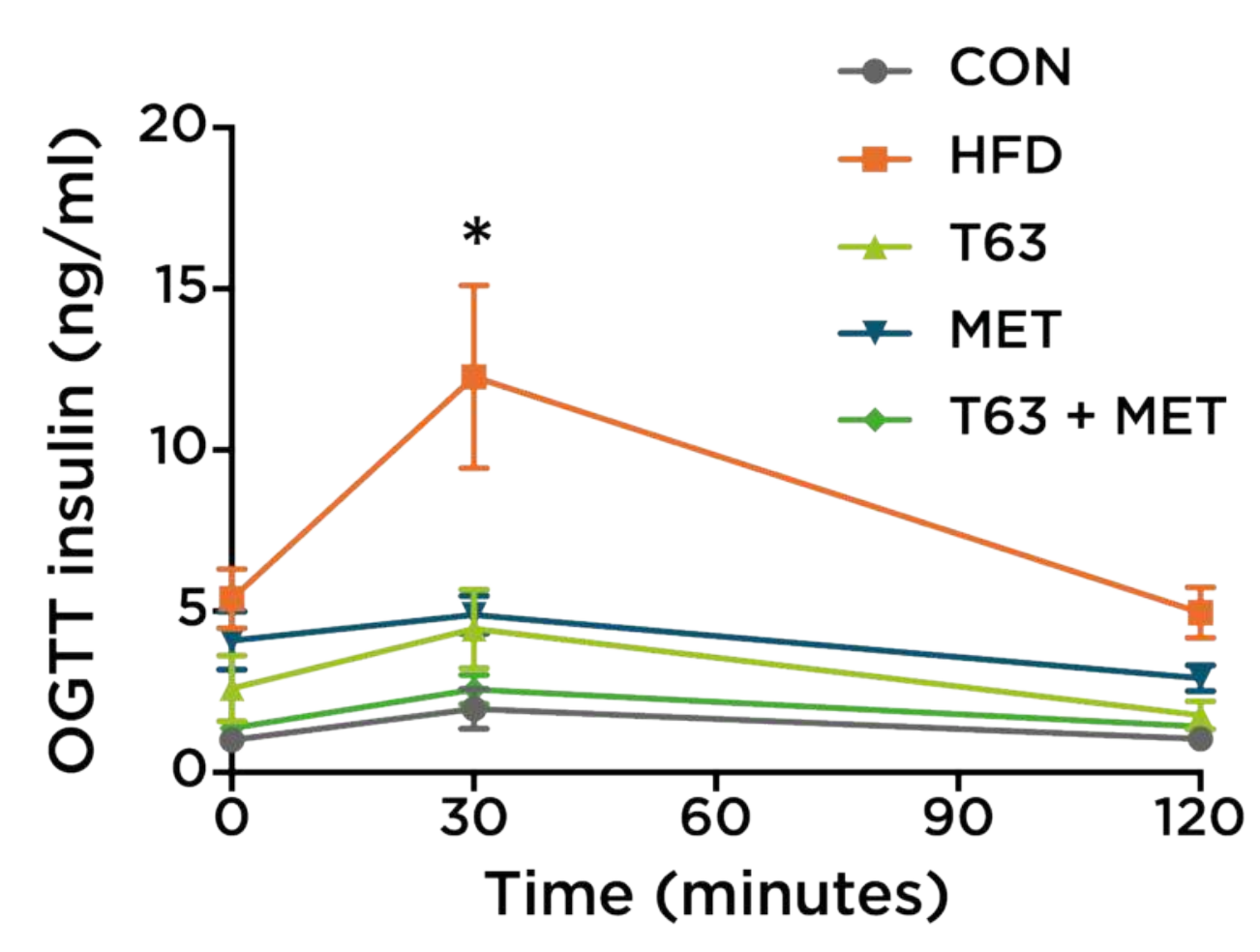

${ }^{*} p<0.05$ vs all groups; ${ }^{*} p<0.05$ vs Control ; $\# p<0.05$ vs HFD
Table 1: anthropometric and biological measurements

\begin{tabular}{|c|c|c|c|c|c|}
\hline & CONTROL & HFD & HFD + T63 & $\begin{array}{c}\text { HFD + } \\
\text { Metformin }\end{array}$ & $\begin{array}{c}\text { HFD + T63 + } \\
\text { Metformin }\end{array}$ \\
\hline Weight (g) & $30.8 \pm 2.0$ & $44.7 \pm 4.6^{*}$ & $33.6 \pm 5.9^{\#}$ & $42.4 \pm 5.6$ & $32.3 \pm 3.7^{\#}$ \\
\hline Fat mass (g) & $4.0 \pm 1.4$ & $16.1 \pm 3.7^{*}$ & $8.3 \pm 4.4^{\#}$ & $12.8 \pm 3.2$ & $7.0 \pm 2.9^{\#}$ \\
\hline $\begin{array}{c}\text { Fasting } \\
\text { Glycaemia } \\
\text { (mg.dl-1) }\end{array}$ & $155.6 \pm 17.3$ & $204.7 \pm 22.9^{*}$ & $188.8 \pm 36.4$ & $217.3 \pm 28.7$ & $185.0 \pm 32.3$ \\
\hline $\begin{array}{c}\text { Fasting plasma } \\
\text { insulin (ng.ml-1) }\end{array}$ & $1.0 \pm 0.3$ & $5.5 \pm 3.4^{*}$ & $1.6 \pm 1.6^{\#}$ & $5.8 \pm 5.2$ & $1.4 \pm 0.5^{\#}$ \\
\hline $\begin{array}{c}\text { HOMA-IR } \\
\text { HOM.I }\end{array}$ & $11.0 \pm 4.9$ & $78.3 \pm 46.2^{*}$ & $23.6 \pm 27.0^{\#}$ & $87.3 \pm 68.0$ & $17.9 \pm 6.3^{\#}$ \\
\hline $\begin{array}{c}\text { Hepatic } \\
\text { triglycerides } \\
\text { (nmol.mg-1) }\end{array}$ & $14.6 \pm 7.5$ & $35.4 \pm 9.3^{*}$ & $21.4 \pm 11.7^{\#}$ & $41.6 \pm 7.1$ & $19.7 \pm 7.2^{\#}$ \\
\hline
\end{tabular}

$* 0<0.05$ vs Control : \#p<0.05 vs HFD

Figure 2: AUC for Insulin measurements during oral glucose tolerance test

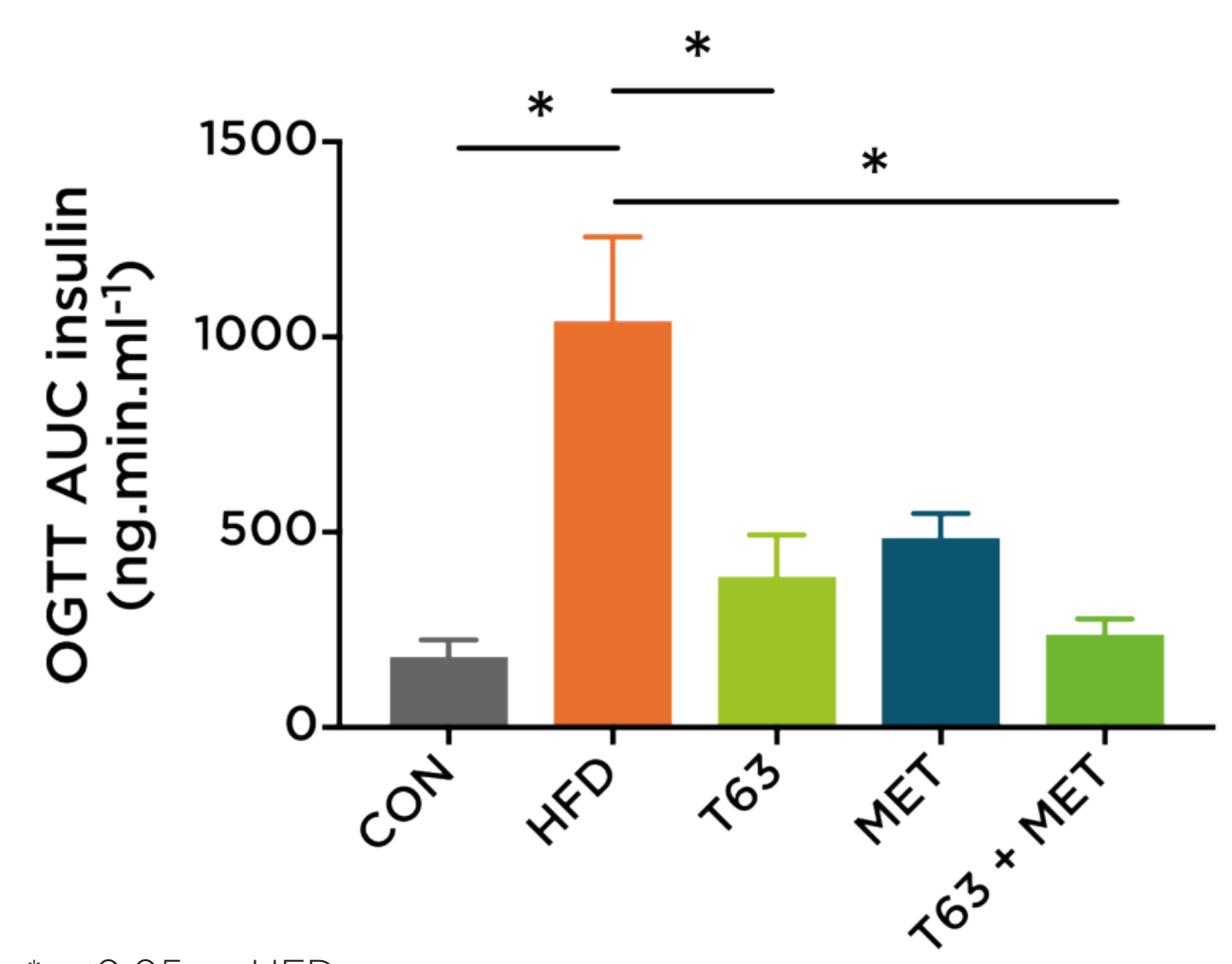

${ }^{*} p<0.05$ vs HFD

Discussion: T63 supplementation was well tolerated and markedly reduced fat mass and body weight gain in HFD-fed mice. This study showed that T63 supplementation is effective to improve insulin sensitivity and reduce hepatic steatosis in HFD-fed mice, with or without concomitant metformin treatment. Moreover, it seems that combination of T63 with metformin does not induce hypoglycemia. Taken together, these first results open the way for a first clinical trial to study this combination in type 2 diabetes. 